\title{
Rheumatoid arthritis-associated gene-gene interaction network for rheumatoid arthritis candidate genes Chien-Hsun Huang ${ }^{1}$, Lei Cong ${ }^{1}$, Jun Xie ${ }^{2}$, Bo Qiao $^{1}$, Shaw-Hwa Lo*1 and Tian Zheng*1
}

\author{
Addresses: ${ }^{1}$ Department of Statistics, Columbia University, 1255 Amsterdam Avenue, $10^{\text {th }}$ Floor, MC44690, New York, New York 10027, USA \\ and ${ }^{2}$ Department of Statistics, 250 North University Street, Purdue University, West Lafayette, Indiana 47907, USA \\ E-mail: Chien-Hsun Huang - ch2526@columbia.edu; Lei Cong - congl@stat.columbia.edu; Jun Xie - junxie@stat.purdue.edu; \\ Bo Qiao - bq2108@columbia.edu; Shaw-Hwa Lo* - slo@stat.columbia.edu; Tian Zheng* - tzheng@stat.columbia.edu \\ *Corresponding author
}

from Genetic Analysis Workshop 16

St Louis, MO, USA 17-20 September 2009

Published: 15 December 2009

BMC Proceedings 2009, 3(Suppl 7):S75 doi: 10.1186/1753-6561-3-S7-S75

This article is available from: http://www.biomedcentral.com/I753-656I/3/S7/S75

(C) 2009 Huang et al; licensee BioMed Central Ltd.

This is an open access article distributed under the terms of the Creative Commons Attribution License (http://creativecommons.org/licenses/by/2.0), which permits unrestricted use, distribution, and reproduction in any medium, provided the original work is properly cited.

\begin{abstract}
Rheumatoid arthritis (RA, MIM I80300) is a chronic and complex autoimmune disease. Using the North American Rheumatoid Arthritis Consortium (NARAC) data set provided in Genetic Analysis Workshop 16 (GAWI6), we used the genotype-trait distortion (GTD) scores and proposed analysis procedures to capture the gene-gene interaction effects of multiple susceptibility gene regions on RA. In this paper, we focused on 27 RA candidate gene regions (53I SNPs) based on a literature search. Statistical significance was evaluated using 1000 permutations. HLADRB I was found to have strong marginal association with RA. We identified I 4 significant interactions $(p<0.01)$, which were aggregated into an association network among 12 selected candidate genes PADI4, FCGR3, TNFRSFIB, ITGAV, BTLA, SLC22A4, IL3, VEGF, TNF, NFKBILI, TRAFI-C5, and MIF. Based on our and other contributors' findings during the GAWI 6 conference, we further studied 24 candidate regions with 336 SNPs. We found 23 significant interactions ( $p$-value $<0.01$ ), nine interactions in addition to our initial findings, and the association network was extended to include candidate genes HLA-A, HLA-B, HLA-C, CTLA4, and IL6. As we will discuss in this paper, the reported possible interactions between genes may suggest potential biological activities of RA.
\end{abstract}

\section{Background}

Rheumatoid arthritis (RA) is a common inflammatory disorder with complex etiology. Although the causes of RA are still unclear, it is believed to be attributed to both genetic and environmental factors. During the last few decades, many new genetic regions have been identified to associate with RA. Remmers et al. [1] showed that STAT4 was an important genetic marker for both RA and systemic lupus erythematosus susceptibility. Kurreeman et al. [2] applied the candidate gene approach to the TRAF1-C5 region and found a polymorphism that increased the susceptibility and severity of RA. Plenge 
et al. [3] studied 14 candidate genes and found significant associations between RA and PTPN22, CTLA4, and PADI4. Although many genes have shown suggestive connections with RA, only HLADRB1 and PTPN22 have been confirmed to increase the genetic risk of developing RA [4]. An explanation for the large number of identified genes might lie in the existence of gene-gene interactions, which, while helpful in identifying and compiling genes, also made the analysis much more complicated.

The traditional approaches used in association studies analyze markers marginally one at a time. As a result, valuable information on the interactions of genes was lost. Zheng et al. [5] proposed an association measure, the genotype-trait distortion (GTD), for evaluating association information on unphased multilocus genotypes from case-control data. GTD was shown to be able to capture interactions between markers that were associated with the disease [5].

Many current association studies are based on dense single-nucleotide polymorphism (SNP) data, with multiple SNPs corresponding to one gene. SNP-based methods are used to identify and replicate the most significant SNP. Without considering the dependence and functional relevance among SNPs within the same gene, most SNP-based association analysis may lead to falsenegative results. As marker density increases, one could consider a gene-based analysis that offers a number of advantages, for instance, taking into account possible multiple disease-associated functional variants within a gene and overcoming the dependence among SNPs due to close proximity. In this study, we used the GTD score and applied a gene-based analysis on two sets of RA candidate genes from the North American Rheumatoid Arthritis Consortium (NARAC) data as part of Problem 1 of the Genetic Analysis Workshop 16 (GAW16).

\section{Methods}

\section{Gene Set I}

The Illumina data set of GAW16 Problem 1 consists of 545,080 SNPs genotyped on 868 RA cases and 1194 unaffected controls from NARAC. In our study, 531 SNPs from 27 candidate gene regions were considered (Table 1). The SNPs numbers and locations were identified by using the SNP mapping information from the National Cancer Institute's Cancer Genetic Markers of Susceptibility (CGEMS) initiative [6]. We included all related SNPs within each gene in the analysis. These 27 candidate genes were selected based on our search of the RA literatures. Among these 27 candidate genes, HLADRB1 and PTPN22 have been widely studied and confirmed to be associated with RA risk. Some of the candidate genes (PADI4, FCRL3, FCGR3, TNFRSF1B, STAT4, CTLA4, IL4, HAVCR1, TNF, MICA, NFKBIL1, OLIG3-TNFAIP3, TRAF1-C5, MHC2TA, and MIF) have been replicated in different populations. A few candidate genes (IL10, BTLA, IL1B, ITGAV, SLC22A4, IL3, VEGF, and RUNX1) showed positive association in individual studies but have not been replicated in other independent data sets. In addition, we also included DLG5 and CARD15 because they were found to be associated with several autoimmune diseases. SNPs with missing data were imputed by fastPhase [7].

\section{Gene Set II}

After the gene $\times$ gene interaction group discussion during the GAW16 conference, 24 candidate genes were selected for further analysis based on the contributors' findings (Table 1), 17 of which were considered in our original analysis (Gene Set I). The seven added genes are IL13, HLA-A, HLA-B, HLA-C, LTA, ILG, and MS4A1, which were identified in other contributors' studies. In other words, we applied our method to re-analyze the same RA data with different candidate genes from Gene Set I.

\section{Methods}

Given $k$ SNP makers, there are $3^{k}$ possible unphased genotypes. We can use the following GTD statistic defined on the sum of squared difference between genotypes' relative frequency among the cases and controls to measure the joint effects of these $k$ SNPs on the disease status, i.e.,

$$
v=\sum_{s=1}^{3^{k}}\left(\frac{n_{D, s}}{n_{D}}-\frac{n_{U, s}}{n_{U}}\right)^{2} .
$$

The statistic $v$ and its variations have been applied successfully in a number of studies [5,8-10]. Specifically, GTD was applied to a candidate gene study of RA during GAW15 [10] and identified significant higher-order interactions that were missed by other methods such as the multifactor dimensionality reduction (MDR) [10-12].

To identify significant interactions, we applied 1000 permutations of the case-control outcomes as in Lo et al. [13]. Based on $v$, we applied the following gene-based analysis procedures developed by Lo et al. [13] to analyze our data sets:

1. Suppose that there are $l$ genes with $m_{I}$ SNPs in gene $i$ and the total number of SNPs is $m\left(\sum m_{i}=m\right)$. For example, $l=27$ and $m=531$ in Gene $e_{1 S e t} \mathrm{I}$. We calculated $v_{i_{d}}$ for SNP $d$ of gene $i(k=1)$ and took an average over the $m_{i}$ SNPs for each gene $i$, which resulted in $l$ averages of the marginal effect of each gene. 
Table I: RA candidate genes

\begin{tabular}{|c|c|c|c|c|}
\hline Gene & Gene Set I & Gene Set II & Locus & SNPs \\
\hline PTPN22 & Yes & Yes & $|p| 3$ & 12 \\
\hline PADI4 & Yes & Yes & Ip36.13 & 18 \\
\hline FCRL3 & Yes & No & $\mid q 21.2-q 22$ & 8 \\
\hline FCGR3 & Yes & Yes & Iq23 & 4 \\
\hline ILIO & Yes & No & $1 q 31-q 32$ & 16 \\
\hline TNFRSFIB & Yes & Yes & Ip36.3-p36.2 & 21 \\
\hline$I L I B$ & Yes & Yes & $2 q 14$ & 7 \\
\hline ITGAV & Yes & Yes & $2 q 31$ & 16 \\
\hline STAT4 & Yes & Yes & $2 q 32.2-q 32.3$ & 30 \\
\hline CTLA4 & Yes & Yes & $2 q 33$ & 5 \\
\hline BTLA & Yes & Yes & $3 q 13.2$ & 6 \\
\hline SLC22A4 & Yes & Yes & $5 q 31$ & 19 \\
\hline $1 L 13$ & No & Yes & $5 q 31$ & 6 \\
\hline IL3 & Yes & Yes & $5 q 31.1$ & 3 \\
\hline IL4 & Yes & No & $5 q 31.1$ & 7 \\
\hline HAVCRI & Yes & No & $5 q 33.2$ & 15 \\
\hline VEGF & Yes & Yes & $6 p 12$ & 12 \\
\hline HLADRBI & Yes & Yes & $6 \mathrm{p} 21.3$ & 5 \\
\hline MICA & Yes & No & $6 p 21.3$ & 32 \\
\hline HLA-A & No & Yes & $6 p 21.3$ & 6 \\
\hline$H L A-B$ & No & Yes & 6p21.3 & 24 \\
\hline HLA-C & No & Yes & 6p21.3 & 29 \\
\hline LTA & No & Yes & $6 \mathrm{p} 21.3$ & 11 \\
\hline NFKBILI & Yes & Yes & $6 \mathrm{p} 21.3$ & 19 \\
\hline$T N F$ & Yes & Yes & $6 \mathrm{p} 21.3$ & 10 \\
\hline OLIG3 - TNFAIP3 & Yes & No & $6 q 23$ & 76 \\
\hline IL6 & No & Yes & $7 q 21$ & 14 \\
\hline TRAFI-C5 & Yes & Yes & $9 q 33-q 34$ & 31 \\
\hline DLG5 & Yes & No & $10 q 23$ & 17 \\
\hline MS4AI & No & Yes & ||$q 3 \mid$ & 13 \\
\hline MHC2TA & Yes & No & $16 p 13$ & 12 \\
\hline CARD I 5 & Yes & No & $16 q 12$ & 11 \\
\hline RUNXI & Yes & No & $21 \mathrm{q} 22.3$ & 104 \\
\hline MIF & Yes & Yes & $22 q 11.2$ & 15 \\
\hline
\end{tabular}

2. We randomly permuted the labels of case $(Y=1)$ and control $(Y=0)$ and repeated Step 1 on the permuted data while the original dependence among SNPs in each gene were retained.

3 . To find the pair-wise interaction among the $l$ genes, we examined $l \times(l-1) / 2$ gene pairs. We first calculated the interaction genotype statistics $v_{i_{d} j_{e}}$ for $m \times(m-1) / 2 \mathrm{SNP}$ pairs $(k=2)$ and defined the SNP-wise interaction as the ratio of incremental interaction versus the maximum of the two marginal effects as in Eq. (2) where " $v$ "stands for maximum of the two values.

$$
r\left(i_{d}, j_{e}\right)=\frac{v_{i_{d} j_{e}}-v_{i_{d}} \vee v_{j_{e}}}{v_{i_{d}} \vee v_{j_{e}}}
$$

Second, we defined the interaction between genes $i$ and $j$ as the average of all SNP-wise ratios in Eq. (3). The ratio is termed as the "mean interaction ratio," Mean-ratio, or $R$ statistic. In addition, we also defined the gene-level "average maximum marginal," or $M$, as in Eq. (4).

$$
\begin{array}{r}
R_{i j}=\frac{\sum_{d=1}^{m_{i}} \sum_{e=1}^{m_{j}} r\left(i_{d}, j_{e}\right)}{m_{i} m_{j}} \\
M_{i j}=\frac{\sum_{d=1}^{m_{i}} \sum_{e=1}^{m_{j}}\left(v_{i_{d}} \vee v_{j_{e}}\right)}{m_{i} m_{j}}
\end{array}
$$

4. Applying the calculation outlined in Step 3 on the permuted data sets, we obtained a set of $l \times(l-1) / 2$ values $\left\{\left(M_{i j}^{(p)}, R_{i j}^{p}\right) ; 1 \leq i<j \leq l\right\}$ for each permutation $p$. The Mean-ratio curve method of the following was applied to identify significant gene-pairs. All $1000 \times l \times(l-1) / 2$ points were put on the $(M, R)$ plane and separated into 100 bins according to the $M$ values. Each bin had $10 \times l \times(l-1) / 2$ points. For each bin, we identified the 99 percentile of the $R$ statistics $\left(R^{*}\right)$ that fell within this bin. By fitting a smoothing spline between the mid-values of each bin $\left(M^{*}\right)$ and $R^{*}$, we constructed the $99 \% R$ threshold curve conditioning on $M$. We identified gene-pairs with observed $R$ statistics above the threshold curve as significant. 
5. In addition to the mean ratio $R$, we used another measure of interaction, the Quantile-ratio statistic. The $Q$ statistic is calculated as the $90^{\text {th }}$ or $95^{\text {th }}$-quantile of the SNP-wise ratios formed by the $m_{i} m_{j}$ SNP pairs. Significant $Q_{i j}$ values were identified by the curve method.

6. An alternative way to evaluate the statistical significance is the Rank method. Similar to Step 4, we have $10 \times$ $l \times(l-1) / 2$ points in each bin. The values of $R$ in each bin are ranked from 1 to $10 \times l \times(l-1) / 2$. For each gene pair, the observed $R$ value received such a rank value $T$, and the $R$ value from permutation $p$ received a rank value $T^{(p)}$. The $p$-value of the gene-pair is then the proportion of $T^{(p)} \geq T$. In this study a significance level of 0.01 is used. The same procedure can also be applied to the $Q$ statistics calculated in Step 5.

\section{Results}

From the average maximum marginal statistics, we observed that all HLADRB1-related gene pairs have the strongest $M$ values. We applied both Mean-ratio and Quantile-ratio methods to capture the interactions. Thirteen interactions are significant at $(p<0.01)$ by the Rank method. Seven interactions were identified by the quantile method at $90^{\text {th }}$ and $95^{\text {th }}$ level. One interaction (ITGAV and VEGF) was identified by the $90^{\text {th }}$-Quantile Rank method. The Curve method identified 10 interactions, all of which were overlapping with that of the Rank method. Table 2 shows the detailed selection results. In
Figure 1, we reported the 14 significant gene-gene interactions from Gene Set I (3.51 significant interactions expected by chance) using red lines.

For Gene Set II (2.76 significant interactions expected by chance), in addition to the interactions identified for Gene Set I (Table 2), another seven interactions were found to be significant by the Mean-ratio Rank method and two additional interactions were found significant by the Curve method. Figure 1 displays the extended networks with these interactions. As seen in Figure 1, MIF seems to be a "hub" in the association network with nine significant interactions. In addition, IL3,VEGF, and $H L A-B$ also have at least five significant interactions.

Morand et al. [14] suggested that MIF is a pivotal mediator of RA. In addition, it has been implicated in many roles such as leukocyte recruitment, activation, and the production of pro-inflammatory cytokines. All of the roles contributed to the pathology of RA and showed the importance of MIF. Many interactions identified in our study have been previously discussed in the literature. It has been demonstrated that serum and synovial fluid levels of MIF were well correlated with the VEGF levels in patients with RA [15]. Nakahara et al. [16] indicated that IL-6 blockade directly suppressed VEGF production in synovial fibroblasts and may consequently reduce serum VEGF levels in patients with RA. Kiriakidis et al. [17] also showed that VEGF production in human macrophages

Table 2: Selected interactions by different proposed procedures $(p$-value $<0.01)$

\begin{tabular}{|c|c|c|c|c|c|}
\hline \multicolumn{3}{|c|}{ Curve method } & \multicolumn{3}{|c|}{ Rank method } \\
\hline Mean Ratio & 90\% Quantile & 95\% Quantile & Mean Ratio & 90\% Quantile & 95\% Quantile \\
\hline $\begin{array}{l}\text { IL3 BTLA } \\
\text { IL3 MIF } \\
\text { IL3 PADI4 } \\
\text { IL3 SLC22A4 } \\
\text { IL3 TNFRSFIB } \\
\text { IL3 TRAFI-C5 } \\
\text { MIF BTLA } \\
\text { MIF FCGR3A } \\
\text { MIF ITGAV } \\
\text { MIF TRAFI-C5 } \\
\text { MIF CTLA4 }\end{array}$ & $\begin{array}{l}\text { IL3 BTLA } \\
\text { IL3 MIF } \\
\text { IL3 PADI4 } \\
\text { IL3 TRAFI-C5 } \\
\text { MIF BTLA } \\
\text { MIF FCGR3A } \\
\text { MIF TRAFI-C5 } \\
\text { FCGR3A } \\
\text { TRAFI-C5 }\end{array}$ & $\begin{array}{l}\text { IL3 BTLA } \\
\text { IL3 MIF } \\
\text { IL3 PADI4 } \\
\text { IL3 TRAFI-C5 } \\
\text { MIF BTLA }\end{array}$ & $\begin{array}{l}\text { IL3 BTLA } \\
\text { IL3 MIF } \\
\text { IL3 PADI4 } \\
\text { IL3 SLC22A4 } \\
\text { IL3 TNFRSFIB } \\
\text { IL3 TRAFI-C5 } \\
\text { MIF BTLA } \\
\text { MIF FCGR3A } \\
\text { MIF HLA-B } \\
\text { MIF ITGAV } \\
\text { MIF TNFRSFIB } \\
\text { MIF TRAFI-C5 } \\
\text { MIF VEGF } \\
\text { NFKBILI HLA-B } \\
\text { NFKBILI VEGF } \\
\text { NFKBILI TNF } \\
\text { VEGF IL6 } \\
\text { VEGF HLA-B } \\
\text { HLA-A HLA-B } \\
\text { HLA-B HLA-C }\end{array}$ & $\begin{array}{l}\text { IL3 MIF }{ }^{\mathrm{a}} \\
\text { IL3 PADI4 } \\
\text { IL3 TRAFI-C5 } \\
\text { ITGAV VEGF } \\
\text { MIF BTLA } \\
\text { MIF FCGR3A } \\
\text { MIF TRAFI-C5 }\end{array}$ & $\begin{array}{l}\text { IL3 BTLA } \\
\text { IL3 MIF } \\
\text { IL3 PADI4 } \\
\text { MIF BTLA } \\
\text { MIF FCGR3A } \\
\text { MIF TRAFI-C5 }\end{array}$ \\
\hline
\end{tabular}

\footnotetext{
${ }^{\text {a }}$ Significant interactions only identified in Gene Set II.
} 


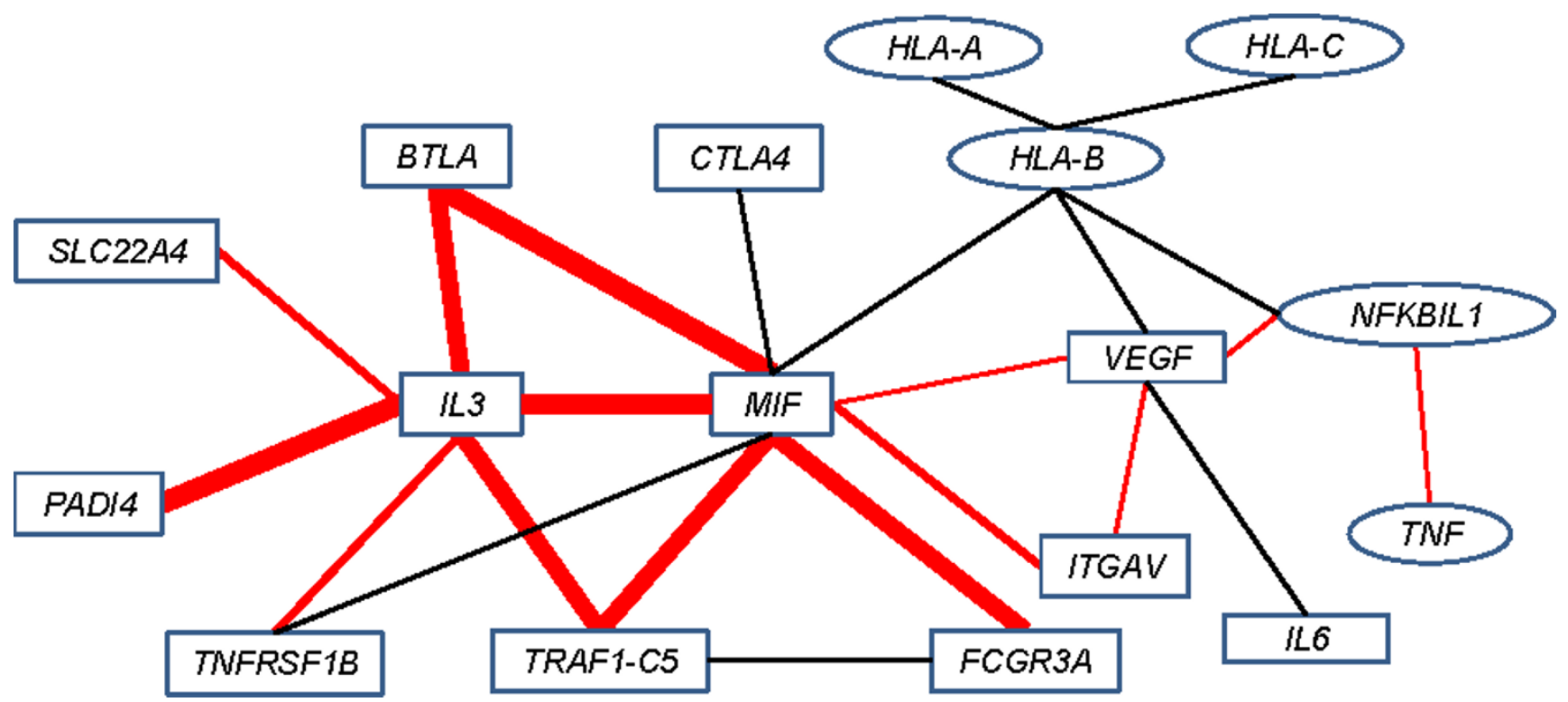

Figure I

Association network with significant interactions identified. All significant interactions identified for Gene Set II are plotted. Interactions in red were also identified in Gene Set I. Different line widths indicate interactions identified by different numbers of proposed procedures.

was NF- $\kappa$ B-dependent, which agreed with our finding of interaction between VEGF and NFKBIL1. VEGF may be directly involved in the activation of RA monocytes and synoviocytes, producing TNF and $I L-6$ via a receptor-coupling event [18]. As noted in Yoo et al. [18], this may imply a direct interaction between VEGF and $I L-6$ or an indirect interaction between VEGF and TNF via NFKBIL1, also found in our results. Onodera et al. [19] demonstrated that MIF enhanced NFKB binding activities of the nuclear extracts from RA synovial fibroblasts. The NFKBIL1, a divergent member of $\mathrm{I}-\kappa$-B proteins that is an inhibitor for NFKB complex, may have potential interaction between MIF and NFKBIL1. In Figure 1, we showed that the indirect connection between MIF and NFKBIL1 via VEGF. Furthermore, the relation between TNF and NFKBdependent signaling pathway of RA patients was discussed by Youn et al. [20].

\section{Conclusion}

In this paper, many relevant candidate regions reported in the literature were chosen for a more detailed analysis. We applied a recently developed method by Lo et al. [13] to identify potential gene-gene interactions that are associated with the susceptibility of RA. First, we found a strong marginal signal between HLADRB1 and RA. In addition, the Rank and the Curve methods based on 1000 permutations identified a number of gene-pairs that are significantly associated with RA, many of which were previously reported in the literature. The association network constructed in our paper may offer additional evidence and insight on gene-gene interactions in the development of RA. Additional experiments and independent data are required to confirm our findings.

\section{List of abbreviations used}

CGEMS: Cancer Genetic Markers of Susceptibility; GAW16: Genetic Analysis Workshop 16; GTD: Genotype-trait distortion; NARAC: North American Rheumatoid Arthritis Consortium; RA: Rheumatoid arthritis; SNP: Single-nucleotide polymorphism.

\section{Competing interests}

The authors declare that they have no competing interests.

\section{Authors' contributions}

$\mathrm{SL}$ and TZ conceived and designed the research. C-HH, $\mathrm{BQ}$, and LC carried out the data analysis and computation. C-HH, JX, S-HL, and TZ analyzed the results and prepared the manuscript. All authors read and approved the final manuscript.

\section{Acknowledgements}

The Genetic Analysis Workshops are supported by NIH grant ROI GM03I575 from the National Institute of General Medical Sciences. The CGEMS SNPs mapping information were requested and accessed with Data Access Committee of the National Cancer Institute permission and 
accessed via the protected portal at http://cgems.cancer.gov/data/. This research was supported by NIH grant ROI GM070789 and NSF grant DMS $07 \mid 4669$.

This article has been published as part of BMC Proceedings Volume 3 Supplement 7, 2009: Genetic Analysis Workshop 16. The full contents of the supplement are available online at $\mathrm{http}: / / \mathrm{www}$.biomedcentral.com/ $\underline{|753-656| / 3 \text { ? issue }=\text { S7. }}$.

\section{References}

I. Remmers EF, Plenge RM, Lee AT, Graham RR, Hom G, Behrens TW, de Bakker PI, Le JM, Lee HS, Batliwalla F, Li W, Masters SL, Booty MG, Carulli JP, Padyukov L, Alfredsson L, Klareskog L, Chen WV, Amos Cl, Criswell LA, Seldin MF, Kastner DL and Gregersen PK: STAT4 and the risk of rheumatoid arthritis and systemic lupus erythematosus. N Engl J Med 2007, 357:977-986.

2. Kurreeman FA, Padyukov L, Marques RB, Schrodi SJ, Seddighzadeh M, Stoeken-Rijsbergen G, Helm-van Mil van der AH, Allaart CF, Verduyn W, Houwing-Duistermaat J, Alfredsson L, Begovich AB, Klareskog L, Huizinga TW and Toes RE: $A$ candidate gene approach identifies the TRAFI/C5 region as a risk factor for rheumatoid arthritis. PLoS Med 2007, 4:e278.

3. Plenge RM, Padyukov L, Remmers EF, Purcell S, Lee AT, Karlson EW, Wolfe F, Kastner DL, Alfredsson L, Altshuler D, Gregersen PK, Klareskog L and Rioux JD: Replication of putative candidategene associations with rheumatoid arthritis in $>4,000$ samples from North America and Sweden: association of susceptibility with PTPN22, CTLA4, and PADI4. Am J Hum Genet 2005, 77:1044-1060.

4. Bowes J and Barton A: Recent advances in the genetics of RA susceptibility. Rheumatology 2008, 47:399-402.

5. Zheng $\mathrm{T}$, Wang $\mathrm{H}$ and Lo $\mathrm{SH}$ : Backward genotype-trait association (BGTA)-based dissection of complex traits in case-control designs. Hum Hered 2006, 62:196-212.

6. Meza JE, Brzovic PS, King MC and Klevit RE: Mapping the functional domains of BRCAI interaction of the ring finger domains of BRCAI and BARDI. J Biol Chem I999, 274:5659-5665.

7. Scheet $P$ and Stephens $M:$ A fast and flexible statistical model for large-scale population genotype data: applications to inferring missing genotypes and haplotypic phase. Am J Hum Genet 2006, 78:629-644.

8. Lo SH and Zheng T: Backward haplotype transmission association (BHTA) algorithm - a fast multiple-marker screening method. Hum Hered 2002, 53:197-215.

9. Lo $\mathrm{SH}$ and Zheng $\mathrm{T}$ : $\mathbf{A}$ demonstration and findings of a statistical approach through reanalysis of inflammatory bowel disease data. Proc Natl Acad Sci USA 2004, 101:10386-10391.

10. Ding $\mathrm{Y}$, Cong L, lonita-Laza I, Lo SH and Zheng T: Constructing gene association networks for rheumatoid arthritis using the backward genotype-trait association (BGTA) algorithm. BMC Proc 2007, I(suppI I):SI3.

II. Ling M, Li X, Yang K, Cui J, Fang B, Guo $X$ and Rotter Il: Evaluating gene $x$ gene and gene $x$ smoking interaction in rheumatoid arthritis using candidate genes in GAWI5. BMC Proc 2007, I (suppl I):SI7.

12. Ritchie MD, Bartlett J, Bush WS, Edwards TL, Motsinger AA and Torstenson ES: Exploring epistasis in candidate genes for rheumatoid arthritis. BMC Proc 2007, I (suppl I):S70.

13. Lo SH, Chernoff H, Cong L, Ding $Y$ and Zheng T: Discovering interaction among BRCAI and other candidate genes associated with sporadic breast cancer. Proc Natl Acad Sci USA 2008, 105:12387-12392

14. Morand EF, Leech M and Bernhagen J: MIF: a new cytokine link between rheumatoid arthritis and atherosclerosis. Nat Rev Drug Discov 2006, 5:399-4I0.

15. Kim HR, Park MK, Cho ML, Yoon CH, Lee SH, Park SH, Leng L, Bucala R, Kang I, Choe J and Kim HY: Macrophage migration inhibitory factor upregulates angiogenic factors and correlates with clinical measures in rheumatoid arthritis. J Rheumatol 2007, 34:927-936.

16. Nakahara $H$, Song J, Sugimoto $M$, Hagihara $K$, Kishimoto $T$, Yoshizaki $\mathrm{K}$ and Nishimoto $\mathrm{N}$ : Anti-interleukin-6 receptor antibody therapy reduces vascular endothelial growth factor production in rheumatoid arthritis. Arthritis Rheum 2003, 48: $152 \mid-1529$.

17. Kiriakidis S, Andreakos E, Monaco C, Foxwell B, Feldmann M and Paleolog E: VEGF expression in human macrophages is NF. kB-dependent: studies using adenoviruses expressing the endogenous NF-kB inhibitor IkBa and a kinase defective form of the IkB kinase 2. J Cell Sci 2003, I 16:665-674.

18. Yoo SA, Bae DG, Ryoo JW, Kim HR, Park GS, Cho CS, Chae CB and Kim WU: Arginine-rich anti-vascular endothelial growth factor (Anti-VEGF) hexapeptide inhibits collagen-induced arthritis and VEGF-stimulated productions of TNF- $\alpha$ and IL-6 by human monocytes. I Immunol 2005, 174:5846-5855.

19. Onodera S, Nishihira J, Koyama $\mathrm{Y}$, Majima T, Aoki $\mathrm{Y}$, Ichiyama H, Ishibashi $T$ and Minami A: Macrophage migration inhibitory factor up-regulates the expression of interleukin-8 messenger RNA in synovial fibroblasts of rheumatoid arthritis patients: common transcriptional regulatory mechanism between interleukin-8 and interleukin-1 $\beta$. Arthritis Rheum 2004, 50: $1437-1447$

20. Youn J, Kim HY, Park JH, Hwang SH, Lee SY, Cho CS and Lee SK: Regulation of TNF-alpha-mediated hyperplasia through TNF receptors, TRAFs, and NF-kB in synoviocytes obtained from patients with rheumatoid arthritis. Immunol Lett 2002, 83:85-93.
Publish with BioMed Central and every scientist can read your work free of charge

"BioMed Central will be the most significant development for disseminating the results of biomedical research in our lifetime."

Sir Paul Nurse, Cancer Research UK

Your research papers will be:

- available free of charge to the entire biomedical community

- peer reviewed and published immediately upon acceptance

- cited in PubMed and archived on PubMed Central

- yours - you keep the copyright
BioMedcentral 